\title{
The Numerical Solutions of Systems of Nonlinear Integral Equations with the Spline Functions
}

\author{
Xiaoyan Liư ${ }^{1}$, Yurong Pan ${ }^{2}$ \\ ${ }^{1}$ University of La Verne, La Verne, USA \\ ${ }^{2}$ Bengbu University, Bengbu, China \\ Email: xliu@laverne.edu, bbxypyr@163.com
}

How to cite this paper: Liu, X.Y. and Pan, Y.R. (2020) The Numerical Solutions of Systems of Nonlinear Integral Equations with the Spline Functions. Journal of $A p$ plied Mathematics and Physics, 8, 470-480. https://doi.org/10.4236/jamp.2020.83037

Received: February 8, 2020

Accepted: March 9, 2020

Published: March 12, 2020

Copyright $\odot 2020$ by author(s) and Scientific Research Publishing Inc. This work is licensed under the Creative Commons Attribution International License (CC BY 4.0).

http://creativecommons.org/licenses/by/4.0/

\section{(c) (i) Open Access}

\begin{abstract}
The main goal of this work is to develop an effective technique for solving nonlinear systems of Volterra integral equations. The main tools are the cardinal spline functions on small compact supports. We solve a system of algebra equations to approximate the solution of the system of integral equations. Since the matrix for the algebraic system is nearly triangular, It is relatively painless to solve for the unknowns and an approximation of the original solution with high precision is accomplished. In order to enhance the accuracy, several cardinal splines are employed in the paper. Our schemes were compared with other techniques proposed in recent papers and the advantage of our method was exhibited with several numerical examples.
\end{abstract}

\section{Keywords}

System of Integral Equations, Nonlinear Integral Equations, Numerical Solutions, Spline Functions

\section{Introduction}

Integral equations appear in many fields, including dynamic systems, mathematical applications in economics, communication theory, optimization and optimal control systems, biology and population growth, continuum and quantum mechanics, kinetic theory of gases, electricity and magnetism, potential theory, geophysics, etc. Many differential equations with boundary-value can be reformulated as integral equations. There are also some problems that can be expressed only in terms of integral equations. Abundant papers have appeared on solving integral equations, for example, Polyanin summarized different solutions 
of integral equations in [1] and [2] [3] published in 2013 and 2016. In [4] [5] and [6], we discussed numerical methods using cardinal splines in solving systems of linear integral equations. In this paper we are going to explore the applications of cardinal splines in solving nonlinear systems of integral equations.

We are interested in the systems of Volterra integral equations of the second kind

$$
\boldsymbol{y}(x)=\boldsymbol{F}\left(\boldsymbol{y}^{\mathrm{T}}(x)\right)+\boldsymbol{g}(x)+\int_{a}^{x} \boldsymbol{K}\left(x, t, \boldsymbol{y}^{\mathrm{T}}(t)\right) \mathrm{d} t,
$$

where the kernel $\boldsymbol{K}\left(x, t, \boldsymbol{y}^{\mathrm{T}}\right)=\left[K_{1}\left(x, t, \boldsymbol{y}^{\mathrm{T}}\right), K_{2}\left(x, t, \boldsymbol{y}^{\mathrm{T}}\right), \cdots, K_{m}\left(x, t, \boldsymbol{y}^{\mathrm{T}}\right)\right]^{\mathrm{T}}$ and $\boldsymbol{g}(x)=\left[g_{1}(x), g_{2}(x), \cdots, g_{m}(x)\right]^{\mathrm{T}}$ are known functions, and $\boldsymbol{y}^{\mathrm{T}}(x)=\left[y_{i}(x)\right]_{i=1}^{m}$ is to be determined; $\boldsymbol{F}\left(\boldsymbol{y}^{\mathrm{T}}(x)\right)=\left[F_{1}\left(\boldsymbol{y}^{\mathrm{T}}(x)\right), F_{2}\left(\boldsymbol{y}^{\mathrm{T}}(x)\right), \cdots, F_{m}\left(\boldsymbol{y}^{\mathrm{T}}(x)\right)\right]$ are known functions in $\boldsymbol{y}^{\mathrm{T}}(x)$.

This paper is divided into six sections. In Section 2 and 3, two univariate cardinal continuous splines on small compact supports are presented. In Section 4, the applications of cardinal splines on solving integral equations are explored. The unknown functions are expressed as linear combinations of horizontal translations of a cardinal spline function. Then a system of equations on the coefficients is deducted. We can solve the system and a good approximation of the original solution is obtained. The sufficient condition for the existence of the inverse matrix is discussed and the convergence is investigated. In Section 5, the numerical examples are given. The non-linear system on unknowns is solved and an accurate approximation of the original solution is obtained in each case. Section 6 contains the concluding remarks.

\section{Cardinal Splines with Small Compact Supports}

Since the paper [7] by Schoenberg published in 1946, spline functions have been studied by many scholars. Spline functions have excellent properties and applications are endless (for example, cf. [8]). The spline functions on uniform partitions are simple to construct and easy to apply, and are sufficient for a variety of applications.

The starting point is frequently the zero degree polynomial B-spline, with the integral iteration formula

$$
\begin{gathered}
B_{0}(x)=\left\{\begin{array}{lc}
1, & -\frac{1}{2}<x<\frac{1}{2}, \\
0, & \text { elsewhere }
\end{array}\right. \\
B_{n}(x)=\int_{-\frac{1}{2}}^{\frac{1}{2}} B_{n-1}(x+t) \mathrm{d} t, n=1,2,3, \cdots
\end{gathered}
$$

we could construct higher order polynomial spline functions with higher degree of smoothness. More specifically, $B_{1}(x)$ has the expression 


$$
B_{1}(x)=\frac{1}{2}|x-1|+\frac{1}{2}|x+1|-|x| .
$$

$B_{n}(x)$ are called one dimensional B-splines, which are polynomial splines and have small supports $\left(-\frac{n+1}{2}, \frac{n+1}{2}\right)$, i.e. $B_{n}(x)=0$ for $x>\frac{n+1}{2}$ or $x<-\frac{n+1}{2}$, and excellent traits (cf. [8]). In my previous papers [4] and [5], low degree orthonormal spline and cardinal splines functions with small compact supports were applied in solving the second kind of Volterra integral equations. In this paper we use the notation $\|u\|_{A}=\max _{x \in A}|u(x)|$.

Let $B_{1, h}(x)=B_{1}\left(\frac{x}{h}\right)$. It is proved that

$$
\sum_{k=-\infty}^{\infty}(a+b k h) B_{1, h}(x-k h)=(a+b x) .
$$

Notice that this particular B-spline is also a cardinal spline, therefore it is straightforward to apply it in interpolations. As far as the convergence rate of interpolation is concerned, we have the following proposition (cf. [9] [10] and [11]).

Proposition 1. Given that $f(x) \in C[a, b], f^{\prime}(x)$ exists and is bounded in $[a, b]$. Let $n$ be an integer, $h=\frac{b-a}{n}$, let $x_{i}=a+i h, f_{i}=f\left(x_{i}\right), i=0,1,2, \cdots, n$,

$$
\Omega f(x)=\sum_{k=0}^{n} f_{k} B_{1, h}(x-k h)
$$

then

$$
\|\Omega f(x)-f(x)\|_{[a, b]} \leq 3\left\|f^{\prime}(x)\right\|_{[a, b]} h^{2} .
$$

If $f(x) \in C(-\infty, \infty)$ and $f^{\prime}(x)$ exists and is bounded, let $h$ be a real number, let $f_{i}=f(i h), i=0,1,2, \cdots$,

$$
\Omega f(x)=\sum_{k=-\infty}^{\infty} f_{k} B_{1, h}(x-k h),
$$

then

$$
\|\Omega f(x)-f(x)\|_{(-\infty, \infty)} \leq 3\left\|f^{\prime}(x)\right\|_{(-\infty, \infty)} h^{2} .
$$

\section{A Univariate $C^{2}$ Cardinal Spline}

By cardinal conditions (cf. [7]), we mean, let $L(x)$ be a function, $\left\{x_{i}\right\}, i=0, \pm 1, \pm 2, \pm 3, \cdots$ be interpolation points, then

$$
L\left(x_{i}\right)=\left\{\begin{array}{ll}
1, & i=0 \\
0, & i \neq 0
\end{array}, \quad i=0, \pm 1,2, \pm 3, \pm 4, \cdots .\right.
$$

The cardinal spline that was originally given in [9] is based on $B_{3}(x)$ from (1.1) using the similar process as in Section 2. Let

$$
L_{3}(x)=6 B_{3}(x)-60 \int_{-\frac{1}{2}}^{\frac{1}{2}} t^{2} B_{2}(x+t) \mathrm{d} t=\int_{-\frac{1}{2}}^{\frac{1}{2}}\left(6-60 t^{2}\right) B_{2}(x+t) \mathrm{d} t .
$$


Then $L_{3}\left(\frac{x}{h}\right)$ satisfies the above cardinal condition when $x_{i}=i h$. Notice that by the construction, $L_{3}(x) \in C^{2}(-\infty, \infty) . \quad L_{3}(x)=0$ for $x \in(-\infty,-2) \cup(2, \infty) . \quad L_{3}(x)$ is a polynomial of degree $\leq 5$ in each subinterval $(-2,-1),(-1,0),(0,1),(1,2)$ of its support. Furthermore, from direct calculation we deduct the following two propositions (cf. [9]).

Proposition 2. Let $L_{3}(x)$ be the cardinal spline constructed above, then

$$
\begin{gathered}
\sum_{k=-\infty}^{\infty} L_{3}\left(\frac{x}{h}-k\right)\left(\alpha(i h)^{2}+\beta(i h)+\gamma\right)=\alpha x^{2}+\beta x+\gamma, \\
\left\|\sum_{k=-\infty}^{\infty} L_{3}\left(\frac{x}{h}-k\right)(i h)^{3}-x^{3}\right\| \leq \frac{1}{6} h^{3},
\end{gathered}
$$

where $\alpha, \beta, \gamma$ are any complex numbers.

Proposition 3. If $f(x) \in C^{3}[a, b]$, let $h=\frac{b-a}{n}, n$ be an integer, let $f_{i}=f(a+i h), i=0,1,2, \cdots$,

$$
\begin{aligned}
\Omega f(x)= & \left(3 f_{0}-3 f_{1}+f_{2}\right) L_{3}\left(\frac{x}{h}+1\right)+\sum_{k=0}^{n} f_{i} L_{3}\left(\frac{x}{h}-k\right) \\
& +\left(3 f_{n}-3 f_{n-1}+f_{n-2}\right) L_{3}\left(\frac{x}{h}-n-1\right) ;
\end{aligned}
$$

then

$$
\|\Omega f(x)-f(x)\|_{[a, b]} \leq 7\left\|f^{\prime \prime \prime}(x)\right\|_{[a, b]} h^{3} .
$$

If $f(x) \in C^{3}(-\infty, \infty)$ and be bounded, let $h$ be a real number, let $f_{i}=f(i h), i=0,1,2, \cdots$,

$$
\Omega f(x)=\sum_{k=-\infty}^{\infty} f_{i} L_{3}\left(\frac{x}{h}-k\right)
$$

then

$$
\|\Omega f(x)-f(x)\|_{(-\infty, \infty)} \leq 7\left\|f^{\prime \prime \prime}(x)\right\|_{(-\infty, \infty)} h^{3} .
$$

\section{Numerical Methods Solving Systems of Integral Equations}

Method 1-V for solving the system of nonlinear Volterra integral equations

As for the Volterra integral Equations (1.1) we solve it in an interval $[a, b]$.

Again we let $h=\frac{b-a}{n}, x_{i}=a+i h, i=0,1, \cdots, n$. Furthermore, by pluging in

$$
\begin{aligned}
& y_{s}(x)=\sum_{k=0}^{n} c_{s, k} B_{1, h}\left(x-x_{k}\right), \\
& K_{s}\left(x, t, y^{\mathrm{T}}\right)=\sum_{i=0}^{n} \sum_{j=0}^{n} K_{s}\left(x_{i}, x_{j}, \boldsymbol{c}_{j}\right) B_{1, h}\left(x-x_{i}\right) B_{1, h}\left(t-x_{j}\right), \\
& g_{s}(x)=\sum_{k=0}^{n} g_{s}\left(x_{k}\right) B_{1, h}\left(x-x_{k}\right), s=1,2, \cdots, m, \text { we get } \\
& \quad \sum_{k=0}^{n} F_{s}\left(\boldsymbol{c}_{k}\right) B_{1, h}\left(x-x_{k}\right)-\sum_{i=0}^{n} \sum_{j=0}^{n} B_{1, h}\left(x-x_{i}\right) \int_{a}^{x} K\left(x_{i}, x_{j}, \boldsymbol{c}_{j}\right) B_{1, h}\left(t-x_{j}\right) \mathrm{d} t \\
& \quad=\sum_{k=0}^{n} g_{s}\left(x_{k}\right) B_{1, h}\left(x-x_{k}\right), s=1,2, \cdots, m
\end{aligned}
$$


Let $x=x_{l}$, we arrive at

$$
\begin{gathered}
F_{s}\left(\boldsymbol{c}_{l}\right)-\sum_{j=0}^{n} K_{s}\left(x_{l}, x_{j}, \boldsymbol{c}_{j}\right) \int_{a}^{x_{l}} B_{1, h}\left(t-x_{j}\right) \mathrm{d} t=g_{s}\left(x_{l}\right), \\
l=0,1,2,3,4, \cdots, n ; s=1,2, \cdots, m
\end{gathered}
$$

which is a simple system of $(n+1) m$ nonlinear equations of unknowns $\left\{c_{s, 0}, c_{s, 1}, \cdots, c_{s, n}\right\}_{s=1,2, \cdots, m}$. Notice that this is a nearly triangular system and it is solvable (the solution may not be unique because it is not linear):

$$
\begin{gathered}
F_{s}\left(\boldsymbol{c}_{0}\right)=g_{s}\left(x_{0}\right), \\
F_{s}\left(\boldsymbol{c}_{1}\right)-\frac{h}{2} \sum_{j=0}^{1} K_{s}\left(x_{1}, x_{j}, \boldsymbol{c}_{j}\right)=g_{s}\left(x_{1}\right), \\
F_{s}\left(\boldsymbol{c}_{l}\right)-\frac{h}{2}\left(K_{s}\left(x_{l}, x_{0}, \boldsymbol{c}_{0}\right)+2 \sum_{j=1}^{l-1} K_{s}\left(x_{l}, x_{j}, \boldsymbol{c}_{j}\right)+K_{s}\left(x_{l}, x_{l}, \boldsymbol{c}_{l}\right)\right)=g_{s}\left(x_{l}\right) \\
l=2,3,4, \cdots, n,
\end{gathered}
$$

where $\boldsymbol{c}_{j}=\left[c_{1, j}, c_{2, j}, \cdots, c_{m, j}\right]^{\mathrm{T}}, j=0,1,2, \cdots, n$.

Proposition 4. Given the Equation (1) and that $f_{s}(x), g_{s}(x) \in C[a, b]$, $f_{s}^{\prime}(x)$ and $g_{s}^{\prime}(x)$ exists and is bounded in $[a, b]$,

$\boldsymbol{K}(x, y, \boldsymbol{u}) \in C^{1}\left([a, b] \times[a, b] \times\left[\alpha_{1}, \beta_{1}\right] \times \cdots \times\left[\alpha_{s}, \beta_{s}\right]\right)$

$\boldsymbol{F}(\boldsymbol{u}) \in C^{1}\left(\left[\alpha_{1}, \beta_{1}\right] \times \cdots \times\left[\alpha_{s}, \beta_{s}\right]\right)$. Furthermore, $\boldsymbol{K}(x, y, \boldsymbol{u})$ and $\boldsymbol{F}(\boldsymbol{u})$ satisfies the condition:

$$
\begin{gathered}
\left|\int_{a}^{b}(\boldsymbol{K}(x, t, \boldsymbol{f}(t))-\boldsymbol{K}(x, t, \boldsymbol{u}(t))) \mathrm{d} t\right|<L M \max _{x \in[a, b]}|\boldsymbol{f}(x)-\boldsymbol{u}(x)| . \\
|| \boldsymbol{F}(\boldsymbol{f}(t))-\boldsymbol{F}(\boldsymbol{u}) \|<\chi \max _{x \in[a, b]}|\boldsymbol{f}(x)-\boldsymbol{u}(x)|
\end{gathered}
$$

where $L M+\chi<1$. Let $n$ be an integer, $h=\frac{b-a}{n}$, let $x_{i}=a+i h$, $f_{s i}=f_{s}\left(x_{i}\right), i=0,1,2, \cdots, n, \quad\left\{c_{s, 0}, c_{s, 1}, \cdots, c_{s, n}\right\}_{s=1}^{m}$ satisfy the nonlinear system $(2.2)$

$$
f_{s}^{*}(x)=\sum_{k=0}^{n} c_{s, i} B_{1, h}(x-k h)
$$

then

$$
\left\|f_{s}^{*}(x)-f_{s}(x)\right\|_{[a, b]}=O\left(h^{2}\right) .
$$

where $\left[f_{1}(x), f_{2}(x), \cdots, f_{m}(x)\right]$ is the exact solution of Equation (1.1).

\section{Method 2-V for solving the Volterra integral equation}

To improve the approximation rate, we apply the spline function $L_{3, h}(x)$. Again we let $h=\frac{b-a}{n}, x_{i}=a+i h, i=0,1, \cdots, n$. Furthermore, let $L_{3, h}(x)=L_{3}\left(\frac{x}{h}\right)$ be the cardinal spline given in Section 3, and

$$
f_{s}(x)=\sum_{k=-1}^{n+1} c_{s, k} L_{3, h}\left(x-x_{k}\right)
$$




$$
\begin{gathered}
K_{s}(x, t, y)=\sum_{i=0}^{n} \sum_{j=0}^{n} K_{s}\left(x_{i}, x_{j}, \boldsymbol{c}_{j}\right) L_{3, h}\left(x-x_{i}\right) L_{3, h}\left(t-x_{j}\right), \\
g_{s}(x)=\sum_{k=0}^{n} g_{s}\left(x_{k}\right) L_{3, h}\left(x-x_{k}\right),
\end{gathered}
$$

$s=1,2, \cdots, m$, where

$$
\begin{gathered}
\boldsymbol{K}\left(x_{-1}, x_{j}, \boldsymbol{c}_{j}\right)=3 \boldsymbol{K}\left(x_{0}, x_{j}, \boldsymbol{c}_{j}\right)-3 \boldsymbol{K}\left(x_{1}, x_{j}, \boldsymbol{c}_{j}\right)+\boldsymbol{K}\left(x_{2}, x_{j}, \boldsymbol{c}_{j}\right), \\
\boldsymbol{K}\left(x_{i}, x_{-1}, \boldsymbol{c}_{j}\right)=3 \boldsymbol{K}\left(x_{i}, x_{0}, \boldsymbol{c}_{j}\right)-3 \boldsymbol{K}\left(x_{i}, x_{1}, \boldsymbol{c}_{j}\right)+\boldsymbol{K}\left(x_{i}, x_{2}, \boldsymbol{c}_{j}\right), \\
\boldsymbol{K}\left(x_{-1}, x_{-1}, \boldsymbol{c}_{j}\right)=3\left(3 \boldsymbol{K}\left(x_{0}, x_{0}, \boldsymbol{c}_{j}\right)-3 \boldsymbol{K}\left(x_{1}, x_{0}, \boldsymbol{c}_{j}\right)+\boldsymbol{K}\left(x_{2}, x_{0}, \boldsymbol{c}_{j}\right)\right) \\
-3\left(3 \boldsymbol{K}\left(x_{0}, x_{1}, \boldsymbol{c}_{j}\right)-3 \boldsymbol{K}\left(x_{1}, x_{1}, \boldsymbol{c}_{j}\right)+\boldsymbol{K}\left(x_{2}, x_{1}, \boldsymbol{c}_{j}\right)\right) \\
+\left(3 \boldsymbol{K}\left(x_{0}, x_{2}, \boldsymbol{c}_{j}\right)-3 \boldsymbol{K}\left(x_{1}, x_{2}, \boldsymbol{c}_{j}\right)+\boldsymbol{K}\left(x_{2}, x_{2}, \boldsymbol{c}_{j}\right)\right), \\
g_{s}\left(x_{-1}\right)=3 g_{s}\left(x_{0}\right)-3 g_{s}\left(x_{1}\right)+g_{s}\left(x_{2}\right) \\
\boldsymbol{K}\left(x_{n+1}, x_{j}, \boldsymbol{c}_{j}\right)=3 \boldsymbol{K}\left(x_{n}, x_{j}, \boldsymbol{c}_{j}\right)-3 \boldsymbol{K}\left(x_{n-1}, x_{j}, \boldsymbol{c}_{j}\right)+\boldsymbol{K}\left(x_{n-2}, x_{j}, \boldsymbol{c}_{j}\right), \\
\boldsymbol{K}\left(x_{i}, x_{n+1}, \boldsymbol{c}_{j}\right)=3 \boldsymbol{K}\left(x_{i}, x_{n}, \boldsymbol{c}_{j}\right)-3 \boldsymbol{K}\left(x_{i}, x_{n-1}, \boldsymbol{c}_{j}\right)+\boldsymbol{K}\left(x_{i}, x_{2}, \boldsymbol{c}_{j}\right), \\
=3\left(3 \boldsymbol{K}\left(x_{n}, x_{n}, \boldsymbol{c}_{j}\right)-3 \boldsymbol{K}\left(x_{n-1}, x_{n}, \boldsymbol{c}_{j}\right)+\boldsymbol{K}\left(x_{n-2}, x_{n}, \boldsymbol{c}_{j}\right)\right) \\
-3\left(3 \boldsymbol{K}\left(x_{n}, x_{n-1}, \boldsymbol{c}_{j}\right)-3 \boldsymbol{K}\left(x_{n-1}, x_{n-1}, \boldsymbol{c}_{j}\right)+\boldsymbol{K}\left(x_{n-2}, x_{n-1}, \boldsymbol{c}_{j}\right)\right) \\
+\left(3 \boldsymbol{K}\left(x_{n}, x_{n-2}, \boldsymbol{c}_{j}\right)-3 \boldsymbol{K}\left(x_{n-1}, x_{n-2}, \boldsymbol{c}_{j}\right)+\boldsymbol{K}\left(x_{n-2}, x_{n-2}, \boldsymbol{c}_{j}\right)\right), \\
g_{s}\left(x_{n+1}\right)=3 g_{s}\left(x_{n}\right)-3 g_{s}\left(x_{n-1}\right)+g_{s}\left(x_{n-2}\right) \\
j=0,1, \cdots, n,
\end{gathered}
$$

where $\boldsymbol{c}_{j}=\left[c_{1, j}, c_{2, j}, \cdots, c_{m, j}\right]^{\mathrm{T}}, j=0,1,2, \cdots, n$. Let $\left\{c_{s, 0}, c_{s, 1}, \cdots, c_{s, n}\right\}_{s=1,2, \cdots, m}$ be unknown coefficients to be determined and $c_{s,-1}=3 c_{s, 0}-3 c_{s, 1}+c_{s, 2}$, $c_{s, n+1}=3 c_{s, n}-3 c_{s, n-1}+c_{s, n-2}$. Plug into the integral Equation (1.1), then we have

$$
\begin{aligned}
& \sum_{k=-1}^{n+1} c_{s, k} L_{3, h}\left(x-x_{k}\right)-\int_{a}^{x} \sum_{i=-1}^{n+1} \sum_{j=-1}^{n+1} K_{s}\left(x_{i}, x_{j}, c_{j}\right) L_{3, h}\left(x-x_{i}\right) L_{3, h}\left(t-x_{j}\right) \mathrm{d} t \\
& =\sum_{k=-1}^{n+1} g_{s}\left(x_{k}\right) L_{3, h}\left(x-x_{k}\right), \quad x \in[a, b] .
\end{aligned}
$$

Let $x=x_{l}$, we arrive at $(l=0,1,2,3,4, \cdots, n)$

$$
c_{s, l}-\sum_{j=-1}^{n+1} K_{s}\left(x_{l}, x_{j}, c_{j}\right) \int_{a}^{x_{l}} L_{3, h}\left(x-x_{i}\right) L_{3, h}\left(t-x_{j}\right) \mathrm{d} t=g_{s}\left(x_{l}\right),
$$

which is still a relatively simple system of equations. For the convergency rate of solution of the Volterra integral Equations (1.1), we have a similar result.

Proposition 5. Given that $f_{s}(x), g_{s}(x) \in C^{3}[a, b], f_{s}^{(4)}(x)$ and $g_{s}^{(4)}(x)$ exists and is bounded in $[a, b]$, $\boldsymbol{K}(x, y, \boldsymbol{u}) \in C^{3}\left([a, b] \times[a, b] \times\left[\alpha_{1}, \beta_{1}\right] \times \cdots \times\left[\alpha_{s}, \beta_{s}\right]\right)$, $\boldsymbol{F}(\boldsymbol{u}) \in C^{3}\left(\left[\alpha_{1}, \beta_{1}\right] \times \cdots \times\left[\alpha_{s}, \beta_{s}\right]\right)$. Furthermore, $\boldsymbol{K}(x, y, \boldsymbol{u})$ satisfies the condition: 


$$
\begin{gathered}
\left\|\int_{a}^{b}(\boldsymbol{K}(x, t, \boldsymbol{f}(t))-\boldsymbol{K}(x, t, \boldsymbol{u}(t))) \mathrm{d} t\right\|<L M \max _{x \in[a, b]}\|\boldsymbol{f}(x)-\boldsymbol{u}(x)\|, \\
\|\boldsymbol{F}(\boldsymbol{f}(t))-\boldsymbol{F}(\boldsymbol{u})\|<\chi \max _{x \in[a, b]}|\boldsymbol{f}(x)-\boldsymbol{u}(x)|
\end{gathered}
$$

where $L M+\chi<1$. Let $n$ be an integer, $h=\frac{b-a}{n}$, let $x_{i}=a+i h$, $f_{s i}=f_{s}\left(x_{i}\right), i=0,1,2, \cdots, n ;\left\{c_{s, 0}, c_{s, 1} \cdots, c_{s, n}\right\}_{s=1}^{m}$ satisfy the system (4.2)

$$
f_{s}^{*}(x)=\sum_{k=-1}^{n+1} c_{s, k} L_{3, h}\left(x-x_{k}\right) \text {, }
$$

then

$$
\left\|f_{s}^{*}(x)-f_{s}(x)\right\|_{[a, b]}=O\left(h^{3}\right) .
$$

where $\left[f_{1}(x), f_{2}(x), \cdots, f_{m}(x)\right]$ is the exact solution of Equation (1.1).

\section{Numerical Examples}

Example 1. Given the system of integral equations

$$
\begin{aligned}
& u(x)+v^{3}(x)-2 x \int_{0}^{x}\left(u(t)+v^{3}(t)\right) \mathrm{d} t=a(x) \\
& v(x)-u^{3}(x)-\frac{1}{5} \int_{0}^{x}\left(u^{3}(t)-v(t)\right) \mathrm{d} t=b(x)
\end{aligned}
$$

where $a(x)=-1 / 2 x^{5}-2 / 3 x^{4}+x^{3}+x^{2}, \quad b(x)=x+1 / 10 x^{2}-x^{6}-1 / 35 x^{7}$, and $[u(x), v(x)]$ are unknown functions.

Let $h=0.1, \quad x_{k}=k h, k=0,1,2, \cdots, 10, u(x)=\sum_{k=0}^{10} c_{k} B_{1, h}\left(x-x_{k}\right)$, $v(x)=\sum_{k=0}^{10} d_{k} B_{1, h}\left(x-x_{k}\right), a(x)=\sum_{k=0}^{10} a\left(x_{k}\right) B_{1, h}\left(x-x_{k}\right)$, $b(x)=\sum_{k=0}^{10} b\left(x_{k}\right) B_{1, h}\left(x-x_{k}\right)$,

$$
K_{s}(x, t, u(t), v(t))=\sum_{i=0}^{10} \sum_{j=0}^{10} K_{s}\left(x_{i}, x_{j}, c_{j}, d_{j}\right) B_{1, h}\left(x-x_{i}\right) B_{1, h}\left(t-x_{j}\right),
$$

we get

$$
\begin{aligned}
& \sum_{k=0}^{n} F_{s}\left(\boldsymbol{c}_{k}\right) B_{1, h}\left(x-x_{k}\right)-\sum_{i=0}^{n} \sum_{j=0}^{n} B_{1, h}\left(x-x_{i}\right) \int_{a}^{x} K\left(x_{i}, x_{j}, \boldsymbol{c}_{j}\right) B_{1, h}\left(t-x_{j}\right) \mathrm{d} t \\
& =\sum_{k=0}^{n} g_{s}\left(x_{k}\right) B_{1, h}\left(x-x_{k}\right), \quad s=1,2, \cdots, m
\end{aligned}
$$

where $c_{j}=\left[c_{1, j}, c_{2, j}\right]=\left[c_{j}, d_{j}\right],\left[g_{1}(x), g_{2}(x)\right]=[a(x), b(x)]$. Let $x=x_{l}$, we arrive at

$$
\begin{gathered}
{\left[c_{0}=a(0), d_{0}=b(0)\right]} \\
{\left[\begin{array}{c}
c_{1}+d_{1}^{3}-\frac{h}{2} \sum_{j=0}^{1} 2 h\left(c_{j}+d_{j}^{3}\right)-a(h)=0 \\
d_{1}-c_{1}^{3}-\frac{h}{2} \sum_{j=0}^{1} \frac{1}{5}\left(-d_{j}+c_{j}^{3}\right)-b(h)=0
\end{array}\right]}
\end{gathered}
$$




$$
\left[\begin{array}{l}
c_{l}+d_{l}^{3}-\frac{h}{2}\left(2(l h)\left(c_{0}+d_{0}^{3}\right)+2 \sum_{j=1}^{l-1} 2(l h)\left(c_{j}+d_{j}^{3}\right)+2(l h)\left(c_{l}+d_{l}^{3}\right)\right)-a(l h)=0 \\
d_{l}-c_{l}^{3}-\frac{h}{2}\left(\frac{1}{5}\left(-d_{0}+c_{0}^{3}\right)+2 \sum_{j=1}^{l-1} \frac{1}{5}\left(-d_{j}+c_{j}^{3}\right)+\frac{1}{5}\left(-d_{l}+c_{l}^{3}\right)\right)-b(l h)=0
\end{array}\right]
$$

Solve the above nonlinear system and we obtain

$\left[c_{0}, c_{1}, c_{2}, c_{3}, c_{4}, c_{5}, c_{6}, c_{7}, c_{8}, c_{9}, c_{10}\right]$

$=[0,0.01004,0.04018,0.09046,0.16091,0.2515$,

$0.36195,0.4921,0.6419,0.81156,1.00125]$

$\left[d_{0}, d_{1}, d_{2}, d_{3}, d_{4}, d_{5}, d_{6}, d_{7}, d_{8}, d_{9}, d_{10}\right]$

$=[0,0.10000,0.20000,0.30001,0.40008,0.50031$,

$0.60084,0.70167,0.80264,0.90365,1.0047]$

Error $<5 \times 10^{-3}$.

(In the paper [12], the error is 0.02579 for $c_{10}$ )

Method 2-V, let $f(x)=u(x)+v^{3}(x), g(x)=v(x)-u^{3}(x)$. The original system becomes

$$
\begin{aligned}
& f(x)-2 x \int_{0}^{x} f(t) \mathrm{d} t=a(x) \\
& g(x)+\frac{1}{5} \int_{0}^{x} g(t) \mathrm{d} t=b(x)
\end{aligned}
$$

Applying $L_{3}$, we still Let $h=0.1, n=10, \quad x_{k}=k h, k=-1,0,1,2, \cdots, 10,11$,

$$
K_{s}(x, t, u(t))=\sum_{k=-1}^{11} \sum_{j=-1}^{11} K_{s}\left(x_{k}, x_{j}, c_{j}, d_{j}\right) L_{3, h}\left(x-x_{k}\right) \cdot L_{3, h}\left(t-x_{j}\right),
$$

$a(x)=\sum_{k=-1}^{11} a\left(x_{k}\right) L_{3, h}\left(x-x_{k}\right), \quad b(x)=\sum_{k=-1}^{11} b\left(x_{k}\right) L_{3, h}\left(x-x_{k}\right)$,

$f(x)=\sum_{k=-1}^{11} c_{k} L_{3, h}\left(x-x_{k}\right), g(x)=\sum_{k=-1}^{11} d_{k} L_{3, h}\left(x-x_{k}\right)$, where

$c_{-1}=f(0)-h f^{\prime}(0)+h^{2} f^{\prime \prime}(0) / 2-h^{3} f^{\prime \prime \prime}(0) / 6=0.01-0.001=0.009$, (we use the given integral equation to find that $f(0)=a(0)=0, f^{\prime}(0)=a^{\prime}(0)=0$,

$\left.f^{\prime \prime}(0)=a^{\prime \prime}(0)=2, \quad f^{\prime \prime \prime}(0)=a^{\prime \prime \prime}(0)=6\right)$. Similarly,

$d_{-1}=g(0)-h g^{\prime}(0)+h^{2} g^{\prime \prime}(0) / 2-h^{3} g^{\prime \prime \prime}(0) / 6=-0.1$. In addition, we let

$c_{n+1}=3 c_{n}-3 c_{n-1}+c_{n-2}$ and $d_{n+1}=3 d_{n}-3 d_{n-1}+d_{n-2}$. Plug into the integral equations, we obtain the nonlinear system:

$$
\begin{aligned}
& \sum_{k=-1}^{11} c_{k} L_{3, h}\left(x-x_{k}\right)-\int_{0}^{x} \sum_{k=-1}^{11} \sum_{j=-1}^{11} 2 x_{k} c_{j} L_{3, h}\left(x-x_{k}\right) L_{3, h}\left(t-x_{j}\right) \mathrm{d} t \\
& =\sum_{k=-1}^{11} a\left(x_{k}\right) L_{3, h}\left(x-x_{k}\right), \\
& \sum_{k=-1}^{11} d_{k} L_{3, h}\left(x-x_{k}\right)+\frac{1}{5} \int_{0}^{x} \sum_{k=-1}^{11} \sum_{j=-1}^{11} d_{j} L_{3, h}\left(x-x_{k}\right) L_{3, h}\left(t-x_{j}\right) \mathrm{d} t \\
& =\sum_{k=-1}^{11} b\left(x_{k}\right) L_{3, h}\left(x-x_{k}\right)
\end{aligned}
$$

the solution of the system is 
$\left[c_{0}, c_{1}, c_{2}, c_{3}, c_{4}, c_{5}, c_{6}, c_{7}, c_{8}, c_{9}, c_{10}\right]$

$=[0,0.011,0.048,0.117,0.224,0.375,0.576$,

0.833,1.152000,1.5389996, 2.000054];

$\left[d_{0}, d_{1}, d_{2}, d_{3}, d_{4}, d_{5}, d_{6}, d_{7}, d_{8}, d_{9}, d_{10}\right]$

$=[0,0.099999,0.199936,0.299270,0.395902,0.484370$,

$0.553336,0.582339,0.537838,0.368533,0.0000508]$.

Furthermore, we solve $u(i h)+v^{3}(i h)=c_{i}, v(i h)-u^{3}(i h)=d_{i}$, $i=0,1,2, \cdots, 10$. The results are

$[u(0), u(h), u(2 h), u(3 h), u(4 h), u(5 h), u(6 h), u(7 h), u(8 h), u(9 h), u(10 h)]$

$=[0,0.010000,0.040000,0.090000,0.160001,0.250003$,

0.360006, 0.490009, 0.640010,0.810010,0.999990],

$[v(0), v(h), v(2 h), v(3 h), v(4 h), v(5 h), v(6 h), v(7 h), v(8 h), v(9 h), v(10 h)]$

$=[0.09999996,0.1999997,0.299999,0.3999978,0.499996$,

$0.5999946,0.699994,0.799995,0.899995,1.000021]$.

The error $<2 \times 10^{-5}$. Our method is much better.

Example 2 Given the system of integral equations

$$
\begin{gathered}
f(x)=a(x)-\frac{1}{2} g^{4}(x)-\frac{1}{2} \int_{0}^{x}\left(\left(-2 x t+3 x^{2}\right) f(t)-t g^{4}(t)\right) \mathrm{d} t \\
g(x)=b(x)-f^{6}(x)-\int_{0}^{x}\left((t-2 x) f^{6}(t)+g(t)\right) \mathrm{d} t
\end{gathered}
$$

where $a(x)=x+\frac{11}{12} x^{4}-\frac{1}{12} x^{6}, b(x)=x+\frac{1}{2} x^{2}+x^{6}-\frac{9}{56} x^{8}$. Let $h=0.05$,

$x_{k}=k h, k=0,1,2, \cdots, 10 . \quad f(x)=\sum_{k=0}^{20} c_{k} B_{1, h}\left(x-x_{k}\right)$,

$g(x)=\sum_{k=0}^{20} d_{k} B_{1, h}\left(x-x_{k}\right)$,

$K_{s}(x, t, u(t), v(t))=\sum_{i=0}^{20} \sum_{j=0}^{20} K_{s}\left(x_{i}, x_{j}, c_{j}, d_{j}\right) B_{1, h}\left(x-x_{i}\right) B_{1, h}\left(t-x_{j}\right)$,

$a(x)=\sum_{k=0}^{20} a\left(x_{k}\right) B_{1, h}\left(x-x_{k}\right), \quad b(x)=\sum_{k=0}^{20} b\left(x_{k}\right) B_{1, h}\left(x-x_{k}\right)$, where

$[f(x), g(x)]$ are unknown functions. we get

$$
\begin{aligned}
& \sum_{k=0}^{n} F_{s}\left(\boldsymbol{c}_{k}\right) B_{1, h}\left(x-x_{k}\right)-\sum_{i=0}^{n} \sum_{j=0}^{n} B_{1, h}\left(x-x_{i}\right) \int_{a}^{x} K\left(x_{i}, x_{j}, \boldsymbol{c}_{j}\right) B_{1, h}\left(t-x_{j}\right) \mathrm{d} t \\
& =\sum_{k=0}^{n} g_{s}\left(x_{k}\right) B_{1, h}\left(x-x_{k}\right), s=1,2, \cdots, m
\end{aligned}
$$

where $c_{j}=\left[c_{1, j}, c_{2, j}\right]=\left[c_{j}, d_{j}\right],\left[g_{1}(x), g_{2}(x)\right]=[a(x), b(x)]$. Let $x=x_{s}$, we arrive at

$$
\begin{gathered}
c_{0}=a(0), d_{0}=b(0) \\
c_{1}+(1 / 2) d_{1}^{4}-a(h)+(h / 2) \sum_{j=0}^{1}(1 / 2)\left(\left(-2(1 h)(j h)+3(1 h)^{2}\right) c_{j}-(j h) d_{j}^{4}\right)=0 \\
d_{1}+c_{1}^{6}-b(h)+(h / 2) \sum_{j=0}^{1}\left(d_{j}+((j h)-2(1 h)) c_{j}^{6}\right)
\end{gathered}
$$




$$
\begin{gathered}
c_{s}+(1 / 2) d_{s}^{4}+(h / 2)\left((1 / 2)\left(\left(-2(s h)(0 h)+3(s h)^{2}\right) c_{0}-(0 h) d_{0}^{4}\right)\right. \\
+2 \sum_{j=1}^{s-1}(1 / 2)\left(\left(-2(s h)(j h)+3(s h)^{2}\right) c_{j}-(j h) d_{j}^{4}\right) \\
\left.+(1 / 2)\left(\left(-2(s h)(s h)+3(s h)^{2}\right) c_{s}-(s h) d_{s}^{4}\right)\right)-a(s h)=0, \\
d_{s}+c_{s}^{6}+(h / 2)\left(\left(d_{0}+((0 h)-2(s h)) c_{0}^{6}\right)+2 \sum_{j=1}^{s-1}\left(d_{j}+((j h)-2(s h)) c_{j}^{6}\right)\right. \\
\left.+\left(d_{s}+((s h)-2(s h)) c_{s}^{6}\right)\right)-b(s h)=0 \\
s=2,3,4, \cdots, 20 .
\end{gathered}
$$

Solve the nonlinear system and we obtain:

$$
\begin{aligned}
& {\left[c_{0}, c_{1}, c_{2}, c_{3}, c_{4}, c_{5}, c_{6}, c_{7}, c_{8}, c_{9}, c_{10}, c_{11}, c_{12}, c_{13}, c_{14}, c_{15}, c_{16}, c_{17}, c_{18}, c_{19}, c_{20}\right]} \\
& =[0,0.5000,0.1000,0.1500,0.2000,0.2500,0.3000,0.3501 \text {, } \\
& 0.4001,0.4501,0.5001,0.5502,0.6002,0.6503,0.7006 \text {, } \\
& \text { 0.7488, 0.7996, 0.8498, 0.8999, 0.94998,1.0000]; } \\
& {\left[d_{0}, d_{1}, d_{2}, d_{3}, d_{4}, d_{5}, d_{6}, d_{7}, d_{8}, d_{9}, d_{10}, d_{11}, d_{12}, d_{13}, d_{14}, d_{15}, d_{16}, d_{17}, d_{18}, d_{19}, d_{20}\right]} \\
& =[0,0.0500,0.1000,0.1500,0.2000,0.2500,0.3000,0.3500 \text {, } \\
& 0.4000,0.4500,0.49999,0.54998,0.59995,0.6499,0.6996 \text {, } \\
& 0.7518,0.8009,0.8507,0.9006,0.9506,1.0006] \text {, } \\
& \text { error }<2 \times 10^{-3}
\end{aligned}
$$

\section{Conclusion}

The proposed method is a simple and effective procedure for solving nonlinear Volterra integral equations of the second kind. The methods can be adapted easily to the Volterra integral equations of the first kind, which have the form $g_{s}(x)=\int_{A} K(x, t) y(t) \mathrm{d} t$, where the upper limit of the integration is a variable. The methods can also be extended to the Fredholm and Volterra integral equations of the first kind or the second kind, where the integral is on an infinite set. The higher degree cardinal splines could also be applied to non-linear integral equations; the resulting system of coefficients will be a little more complicated non-linear systems, which takes more time and effort to solve. Compared with the recent paper [2], our method is more effective.

\section{Acknowledgements}

The research is partially supported by the research funds of the University of La Verne, and by key natural science foundation of Anhui education commission (NO.KJ2017A568), natural science foundation of Bengbu University (NO.2018CXY045; 00009134).

\section{Conflicts of Interest}

The authors declare no conflicts of interest regarding the publication of this paper. 


\section{References}

[1] Polyanin, A.D. (1998) Handbook of Integral Equations. CRC Press LLC, New York. https://doi.org/10.1201/9781420050066

[2] Singh, I. and Kumar, S. (2016) Haar Wavelet Method for Some Nonlinear Volterra Integral Equations of the First Kind. Journal of Computational and Applied Mathematics, 292, 541-552. https://doi.org/10.1016/j.cam.2015.07.022

[3] Aziz, I. and Siraj-ul-Islam (2013) New Algorithms for the Numerical Solution of Nonlinear Fredholm and Volterra Integral Equations Using Haar Wavelets. Journal of Computational and Applied Mathematics, 239, 333-345. https://doi.org/10.1016/j.cam.2012.08.031

[4] Liu, X. and Xie, J. (2014) Numerical Methods for Solving Systems of Fredholm Integral Equations with Cardinal Splines. AIP Conference Proceedings, 1637, 590. https://doi.org/10.1063/1.4904628

[5] Liu, X., Liu, Z. and Xie, J. (2015) Solving Systems of Volterra Integral Equations with Cardinal Splines. Journal of Applied Mathematics and Physics, 3, 1422-1430. https://doi.org/10.4236/jamp.2015.311170

[6] Liu, X., Xie, J., Liu, Z. and Huang, J. (2020) The Cardinal Spline Methods for the Numerical Solution of Nonlinear Integral Equations. Journal of Chemistry, 2020, Article ID: 3236813. https://doi.org/10.1155/2020/3236813

[7] Schoenberg, I.J. (1946) Contributions to the Problem of Approximation of Equidistant Data by Analytic Functions. Quarterly of Applied Mathematics, 4.

[8] Chui, C. K. (1988) Multivariate Splines. SIAM, Philadelphia, PA. https://doi.org/10.1137/1.9781611970173

[9] Liu, X. (2006) Univariate and Bivariate Orthornormal Splines and Cardinal Splines on the Compact Supports. Journal of Computational and Applied Mathematics, 195, 93-105. https://doi.org/10.1016/j.cam.2005.04.070

[10] Liu, X. (2007) Interpolation by Cardinal Exponential Splines. The Journal of Information and Computational Science, 4, 179-194.

[11] Liu, X. (2007) Interpolation by Cardinal Trigonometric Splines. International Journal of Pure and Applied Mathematics, 40, 115-122.

[12] Biazar, J., Babolian, E. and Islam, R. (2003) Solution of a System of Volterra Integral Equations of the First Kind by Adomian Method. Applied Mathematics and Computation, 139, 249-258. https://doi.org/10.1016/S0096-3003(02)00173-X 